\title{
Did the SARS-CoV-2 effect pregnancy complications?
}

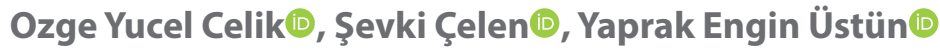 \\ University of Health Sciences Etlik Zübeyde Hanım Women's Health Care, Training and Research Hospital, Ankara, Turkey
}

\begin{abstract}
Objectives: This study aimed to evaluate the effect of SARS-CoV-2 on pregnancy complications, which increased compared to the same period before the pandemic.

Material and methods: This prospective study was conducted at Etlik Zübeyde Hanım Womenss Health Practices \& Research Center between June 2020 and July 2020. The asymptomatic term PROM and miscarriage pregnant women were screened for SARS-CoV-2 (severe acute respiratory syndrome coronavirus-2) positivity by using IgM and IgG antibody tests. All the positive cases were confirmed by real-time reverse transcriptase-polymerase chain reaction (RT-PCR). All the patients answered 12 questions to determine their approach to COVID-19.

Results: Four of the 205 (2\%) term PROM patients had positive anti-SARS-CoV-2 IgM antibody tests, one of the 205 (0.5\%) term PROM patients had a positive anti-SARS-CoV- 2 lgG antibody test, and one of the 205 (\% 0.5) term PROM patients had positive anti-SARS-CoV-2 IgM and lgG antibody tests. All 230 miscarriage patients had negative lgM/lgG antibody tests. Four of the positive antibody cases had positive RT-PCR tests $(2 \%)$ and were referred to a pandemic hospital. The patients thought that $93.2 \%$ and $95.6 \%$ of pregnant women with term PROM and miscarriage, respectively, SARS-CoV- 2 would infect themselves, and $89.8 \%$ and $92.6 \%$, respectively, would infect their children. The number of patients who would not consider pregnancy if they were not pregnant during this period was significantly higher in the term PROM (48.3\%) compared to the miscarriage $(27.8 \%)$ patients $(p<0.005)$.
\end{abstract}

Conclusions: No COVID-19 cases were determined in the asymptomatic miscarriage patients. In the asymptomatic term PROM patients, the effects of COVID were not observed.

Key words: COVID-19; asymptomatic; pregnancy; miscarriage; term prelabor rupture of membranes

Ginekologia Polska 2021; 92, 12: 872-877

\section{INTRODUCTION}

SARS-CoV-2, an enveloped single-stranded RNA virus, has spread all over the world since late 2019 , affecting millions, causing the coronavirus disease 19 (COVID-19) pandemic $[1,2]$. The virus may be transmitted through droplets and hand contact with contaminated surfaces and eventually reach nasal mucosa, eyes, and the mouth $[3,4]$. The symptoms of COVID-19 are similar in pregnant and non-pregnant women, with common coughing but fewer headaches and fevers $[5,6]$. Its clinical course is similar to that in non-pregnant women, according to several sources. However, the Centers for Disease Control and Prevention (CDC) surveillance reports the need for increased intensive care and mechanical ventilation in pregnant women) $[5,7,8]$.

Different policies (personal, social, and health measures) have been followed across countries to minimize the transmission of COVID-19, but the number of asymptomatic cases as a result of these policies is still not clear [2,9]. However, in different studies, SARS-CoV-2 positivity has been reported to have a wide range (13.7-71\%) in asymptomatic pregnant cases $[7,8,10]$.

The vertical transmission of COVID-19 is limited to a few cases, and it has been shown that most newborns become infected later [11-13]. However, the maternal, early, and late fetal consequences of COVID-19's infectivity are still unknown. Although it is associated with preterm and cesarean symptomatic cases, pregnancy outcomes in asymptomatic pregnant women are still unclear [14].

Early and effective measures have been taken in Turkey with the guidelines formed by the Ministry of Health [3]. However, the number of asymptomatic cases is undeniable, and though it is unknown whether an increase in pregnancy complications may develop in asymptomatic pregnant women during this period, a significant increase in complications has been observed for term PROM and miscarriage patients compared to last year [15]. To deter-

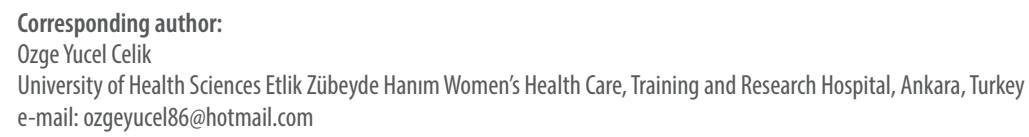


mine the asymptomatic COVID-19 cases and their possible relationship, a SARS-CoV-2 antibody tests were performed in these pregnancy complication cases, and they increased accordingly. This study aimed to evaluate the effect of SARS-CoV-2 in terms of prelabor rupture of membrane (PROM) and miscarriage.

\section{MATERIAL AND METHODS}

Our hospital is a tertiary reference center for obstetrics and gynecology. This study was approved by both Turkish Ministry of Health and the institutional ethics committee with Decision Number: 2020/73. Our study data included pregnant women who were admitted for term PROM and miscarriage without viral symptoms between June 2020 and July 2020. During the pandemic period, our hospital continued to perform monitoring and deliveries of asymptomatic pregnant women without a diagnosis of COVID-19. In this period, routine COVID-19 screening was not performed for the pregnant women; however, all the patients were evaluated in detail regarding symptoms, contact with COVID-19 patients, and history of foreign travels. The patients with suspected COVID-19 were isolated if they needed close follow-up, and if the SARS-CoV-2 PCR result was negative, the hospitalization continued, and patients with positive results were referred to pandemic hospitals. Social distance was provided between patients during the hospitalization of all patients, and the use of masks was mandatory.

SARS-CoV-2 antibody tests were performed in patients diagnosed with term PROM and miscarriage, and blood samples were routinely taken. SARS-CoV-2 IgM and IgG antibodies from the serum samples were tested by the COVID-19 lgM/lgG Rapid Test Device (COV-W23M, ECOTEST, Assure Tech. Co. Ltd., Hangzhou, China). The COViD-19 lgM/lgG rapid antibody test is a lateral flow immunoassay to detect anti-SARS-CoV-2 lgM/lgG. The venous blood specimens are drawn by disposable pipette and dropped into a test device; three drops are then added followed by a 15-minute wait time. If the specimens are stored for a long time, they may be kept at $2-8^{\circ} \mathrm{C}$ for up to seven days and may not be allowed to freeze. The kits have three colored lines [control (C) region IgM region, and Ig G region]. If the colored line in the $C$ region changes from blue to red and the colored line shows in the lgM and/or lgG test region, the result is positive for COVID-19 virus specific-IgM and/or lgG antibodies. If the colored line in the $\mathrm{C}$ region changes from blue to red and no line shows in the IgM and/or IgG test region, the result is negative. If the colored line $C$ region fails to change, the tests are invalid.

Anti-SARS-CoV-2 reverse-transcription polymerase chain reaction (RT-PCR) was tested from nasopharyngeal and oropharyngeal samples in the patients with a positive lgM antibody test. The samples were transported within 12 hours.
The transported samples were assessed using the COVID-19 RT-qPCR detection kit (IVD-CE) (Bioeksen, Istanbul, Turkey) at a molecular virology laboratory. The rapid diagnosis was achieved with the kit via a one-step reverse transcription (RT) and real-time PCR (qPCR) (RT-qPCR) targeting the SARS-CoV-2 (2019-nCoV)-specific RdRp (RNA-dependent RNA polymerase) gene fragment.

The prospective study required written informed consent, which was obtained from all the patients before they participated. Twelve questions were asked to all the patients to determine their level of knowledge and opinions about COVID-19. PROM is defined as the rupture of the membrane after the $37^{\text {th }}$ gestational week before regular contractions occur; miscarriage is a spontaneous abortion leading to the loss of complete or incomplete pregnancy before the $22^{\text {nd }}$ week of gestation or with a fetal weight less than $500 \mathrm{~g}$. The early pregnancy assessment unit (EPAU) is a service that monitors pregnancy complications before the $22^{\text {nd }}$ gestational week. Patients over 45 and those with the following conditions were excluded from the study: current pregnancy with previous miscarriage, multiple miscarriages, specified infectious disease, diabetes, obesity (BMI $\geq 35$ ), thyroid disease, thrombophilia, teratogen drug use, pregnancy trauma, uterine anomaly, and known chromosomal and/or structural anomalies. Data entry errors and whether the parameters were within the expected range were checked before the statistical analysis. Normality assumptions of the continuous variables were examined using the skewness and kurtosis coefficients, a histogram, Q-Q plots, and the Kolmogorov-Smirnov test. The relationships between the categorical variables were tested by chi-square analysis. The descriptive analyses and categorical variables were defined as numbers and percentages. The numerical variables were defined as median (interval) or mean \pm standard deviation. All analyses were performed using IBM SPSS software version 23 . Any $p$-values less than 0.05 were considered statistically significant.

\section{RESULTS}

During the study period, 462 SARS-CoV-2 antibody test kits were used in total; 27 kits were excluded because of repeated and invalid results. Totally, 205 term PROM and 230 miscarriage patients participated. The demographic features and clinical characteristics of the term PROM and miscarriage pregnancy groups are shown in Table 1.

Four of the 205 (2\%) term PROM patients had positive anti-SARS-CoV-2 IgM antibody tests, one of the 205 (0.5\%) term PROM patients had a positive anti-SARS-CoV-2 lgG antibody test, and one of the 205 (0.5\%) term PROM patients had positive anti-SARS-CoV-2 IgM and IgG antibody tests. All 230 miscarriage patients had negative lgM/lgG antibody tests. Four of the positive antibody cases had positive 
Table 1. Demographic features and clinical characteristics in term PROM and miscarriage pregnancy groups

\begin{tabular}{|c|c|c|}
\hline & $\begin{array}{l}\text { Term PROM } \\
(\mathrm{n}=205)\end{array}$ & $\begin{array}{l}\text { Miscarriage } \\
(n=230)\end{array}$ \\
\hline Age & $26(16-43)$ & $29(16-30)$ \\
\hline Gravity & $2(1-7)$ & $2(1-9)$ \\
\hline Parity & $1(0-6)$ & $1(0-8)$ \\
\hline BMI & $29.5 \pm 5.3$ & $25 \pm 2.9$ \\
\hline High risk pregnancy & $16(7.9 \%)$ & 0 \\
\hline Gestational diabetes mellitus & $6(3 \%)$ & \\
\hline Hypertension & $4(2 \%)$ & \\
\hline Intrauterine growth retardation & $5(2.4 \%)$ & \\
\hline Familial Mediterranean fever & $1(0.5 \%)$ & \\
\hline \multicolumn{3}{|l|}{ Education } \\
\hline Uneducated & $25(12.2 \%)$ & $24(10.4)$ \\
\hline Primary school & $80(39 \%)$ & $50(21.7 \%)$ \\
\hline Secondary school & $24(11.7 \%)$ & $28(12.2)$ \\
\hline High school & $55(26.8 \%)$ & $103(44.8 \%)$ \\
\hline University & $21(10.2 \%)$ & 25 (10.9\%) \\
\hline \multicolumn{3}{|l|}{ Nationality } \\
\hline Turkish Republic & $164(80 \%)$ & $196(85.2 \%)$ \\
\hline Syria & $26(12.7 \%)$ & $24(10.4 \%)$ \\
\hline Iraq & $10(4.9 \%)$ & $5(2.2 \%)$ \\
\hline Others & $5(2.4 \%)$ & $5(2.2 \%)$ \\
\hline \multicolumn{3}{|l|}{ Socioeconomic status } \\
\hline Low & $79(38.5 \%)$ & $72(31.3 \%)$ \\
\hline Moderate/High & $126(61.5 \%)$ & $158(68.7 \%)$ \\
\hline Gestational week & $38(37-41)$ & $9(6-21)$ \\
\hline \multicolumn{3}{|l|}{ Delivery } \\
\hline Spontaneous vaginal birth & $98(48.8 \%)$ & \\
\hline Cesarean section & $103(51.2 \%)$ & \\
\hline Anti-SARS-CoV-2 IgM positivity & $4(2 \%)$ & 0 \\
\hline Anti-SARS-CoV-2 IgG positivity & $1(0.5 \%)$ & 0 \\
\hline $\begin{array}{l}\text { Anti-SARS-CoV-2 lgM and lgG } \\
\text { positivity }\end{array}$ & $1(0.5 \%)$ & 0 \\
\hline SARS-CoV-2 RT-PCR positivity & $4(2 \%)$ & 0 \\
\hline
\end{tabular}

PROM — prelabor rupture of membrane; BMI — body mass index

RT-PCR tests (2\%) and were referred to a pandemic hospital. All the positive cases were diagnosed during the last week of the study.

The first case was a 37-year-old multiparous woman with gestational diabetes mellitus; her BMI was $29.2 \mathrm{~kg} / \mathrm{m}^{2}$, and she delivered by caesarean section at the $37^{\text {th }}$ gestational week because of fetal distress. She was followed up seven days in the hospital without symptoms. After she was discharged, she had a positive anti-SARS-CoV-2 IgM test, but she did not accept a RT-PCR test. The second case was a 34-year-old primigravid woman; her BMI was $27.3 \mathrm{~kg} / \mathrm{m}^{2}$, and she de- livered by caesarean section at the 40th gestational week because of cephalopelvic disproportion. The third case was a 34-year-old multiparous woman; her BMI was $26.2 \mathrm{~kg} / \mathrm{m}^{2}$, and she delivered by caesarean section at the 38 th gestational week because of a previous caesarean delivery. The fourth case was a 31-year-old multiparous woman; her BMI was $22 \mathrm{~kg} / \mathrm{m}^{2}$, and she delivered by caesarean section at the $37 \mathrm{th}$ gestational week because of a previous caesarean delivery. After positive RT-PCR tests, three of the patients were referred to a pandemic hospital. The fifth case was a 19-year-old, primigravid woman, her BMI was $42.2 \mathrm{~kg} / \mathrm{m}^{2}$, and she delivered by caesarean section at the $37^{\text {th }}$ gestational week because of a breech presentation. She had positive anti-SARS-CoV-2 IgM and IgG antibody tests. After a positive RT-PCR test, she was referred to a pandemic hospital.

The answers given by the patients to the 12 questions are shown in Table 2. All the patients had information about COVID-19. The patients thought that $93.2 \%$ and $95.6 \%$ of pregnant women with term PROM and miscarriage, respectively, SARS-CoV-2 would infect themselves, and $89.8 \%$ and $92.6 \%$, respectively, would infect their children. The number of patients who thought that hospital precautions were not taken was significantly higher in terms of PROM (11.2\%) than miscarriage $(5.2 \%)$ pregnancy $(p=0.022)$ patients. The number of patients who do not intend to vaccinate their child if the COVID-19 vaccine is developed was significantly higher in the term PROM (10.7\%) than in the miscarriage (5.2\%) $(p=0.032)$ participants.

The number of patients who would not consider pregnancy if they were not pregnant during this period was significantly higher in the term PROM (48.3\%) compared to the miscarriage $(27.8 \%)(p<0.005)$ participants.

\section{DISCUSSION}

Although common precautions are taken for SARS-CoV-2 in the world, screening and follow-up programs vary between countries. No symptomatic or COVID-19 patient was admitted to our hospital during the pandemic period. The number of total asymptomatic cases is unclear because no specific screening program exists for pregnancy. According to the data from the New York City hospitals where the patients who applied for delivery were screened, $33(13.7 \%)$ of 210 asymptomatic patients were observed to be COVID-19 positive [7]. Despite the small number of patients, SARS-CoV-2 positivity was reported in $10(71 \%)$ of the 14 asymptomatic pregnant women, and symptoms began to be observed in the patients during the delivery period [8]. Another study reported that 22 (2.9\%) of 770 pregnant women who were screened at their application for delivery had asymptomatic COVID-19 positivity [16]. Nine (1.2\%) of 757 patients hospitalized for deliveries had asymptomatic COVID-19; none of the patients had symptoms at the de- 
Table 2. The patient's knowledge and opinions about COVID-19

\section{Questions}

Do you have any information about COVID-19?

If yes, what is the source?

TV-Internet

Family-Neighbor

Hospital

Do you think COVID-19 can infect you?

If yes, from whom do you think it can be transmitted?

Family

Hospital

Neighbor

Co-worker

Do you think SARS- can infect your children?

If yes, from whom do you think it can be transmitted?

Family

Hospital

School

Are you taking precautions?

If yes, please specify

Hygiene

Social distance

Mask

Do you find hospital precautions sufficient?

If no, why?

Crowed

Hygiene problems

Careless patients

Have you considered vaccinating your child during the pandemic?

If the COVID-19 vaccine is developed, will your child be vaccinated?

If no, why?

Undecided

Not trust

If you were not pregnant, would you consider pregnancy during a pandemic?

Do family elders live with you?

If yes, what are the precautions?

Hygiene

Social distance

Mask

livery period [17]. Considering the high false-negative rates of diagnostic tests [18], it is thought that asymptomatic patients are more common. In previous studies, pregnant women hospitalized for delivery were screened regardless of the diagnosis. We compared pregnancy complications that occurred during the pandemic period and in

\begin{tabular}{|c|c|c|c|c|}
\hline \multicolumn{2}{|c|}{ Term PROM (n: 205) } & \multicolumn{2}{|c|}{ Miscarriage (n: 230) } & \multirow{2}{*}{ p } \\
\hline Yes & No & Yes & No & \\
\hline $205(100 \%)$ & $0(0 \%)$ & $230(100 \%)$ & $0(0 \%)$ & 1 \\
\hline $177(86.3 \%)$ & & 195 (84.8\%) & & \\
\hline $20(9.8 \%)$ & & $29(12.6 \%)$ & & \\
\hline $8(3.9 \%)$ & & $6(2.6 \%)$ & & \\
\hline $191(93.2 \%)$ & $14(6.8 \%)$ & $220(95.6 \%)$ & $10(4.4 \%)$ & 0.296 \\
\hline $116(60.7 \%)$ & & $133(60.5 \%)$ & & \\
\hline $20(10.5 \%)$ & & $12(5.5 \%)$ & & \\
\hline $24.00(12.6 \%)$ & & $8(8.2 \%)$ & & \\
\hline $31.00(16.2 \%)$ & & 57 (25.9\%) & & \\
\hline $184(89.8 \%)$ & $21(10.2 \%)$ & $213(92.6 \%)$ & $17(7.4 \%)$ & 0.293 \\
\hline $139(75.5 \%)$ & & 167 (78.4\%) & & \\
\hline $21(11.4 \%)$ & & $13(6.1 \%)$ & & \\
\hline $24(13.1 \%)$ & & $33(15.5 \%)$ & & \\
\hline 205 (100\%) & $0(0 \%)$ & $230(100 \%)$ & $0(0 \%)$ & 1 \\
\hline 68 (33.2\%) & & $66(28.7 \%)$ & & \\
\hline $16(7.8 \%)$ & & $12(5.2 \%)$ & & \\
\hline $121(59 \%)$ & & $152(66.1 \%)$ & & \\
\hline $182(88.8 \%)$ & $23(11.2 \%)$ & $218(94.8 \%)$ & $12(5.2 \%)$ & 0.022 \\
\hline & $7(30.4 \%)$ & & $2(16.7 \%)$ & \\
\hline & $3(13.1 \%)$ & & $2(16.7 \%)$ & \\
\hline & $13(56.5 \%)$ & & $8(66.6 \%)$ & \\
\hline 204 (99.5\%) & $1(0.5 \%)$ & $0(0 \%)$ & $230(100 \%)$ & 0.471 \\
\hline $183(89.3 \%)$ & $22(10.7 \%)$ & $218(94.8 \%)$ & $12(5.2 \%)$ & 0.032 \\
\hline & $11(50 \%)$ & & $3(25 \%)$ & \\
\hline & $11(50 \%)$ & & $9(575)$ & \\
\hline $106(51.7 \%)$ & $99(48.3 \%)$ & $166(72.2 \%)$ & $64(27.8 \%)$ & $<0.005$ \\
\hline $35(15.2 \%)$ & $195(84.8 \%)$ & $34(16.6 \%)$ & 171 (83.4\%) & 0.694 \\
\hline $27(77.1 \%)$ & & $25(73.5 \%)$ & & \\
\hline $5(14.3 \%)$ & & $5(14.7 \%)$ & & \\
\hline $3(8.6 \%)$ & & $4(11.8 \%)$ & & \\
\hline
\end{tabular}

the same period last year, and the rates of term PROM and miscarriage increased statistically and significantly [15]. The term PROM and miscarriage pregnancies were scanned by SARS-CoV-2 lgM/lgG antibody and RT-PCR tests.

COVID-19 has been associated with preterm labor and caesarean delivery in symptomatic pregnancy [14]. 
SARS-CoV-2 positivity was reported in 22 (29.3\%) of 75 asymptomatic pregnant women, but these patients were not associated with maternal adverse outcomes and preterm labor [19]. Pregnant women in the pro-inflammatory period in the early and late weeks are expected to reveal serious inflammatory results due to the cytokine storm caused by COVID-19 in symptomatic patients [20]. The fact is that the inflammatory process is still unknown in asymptomatic cases, but inflammation shows a strong correlation with the early rupture of membranes [21]. In our study, five of 205 (2.5\%) term PROM had IgM positive, and four of 205 (2\%) term PROM patients were RT-PCR positive. According to health ministry data from Turkey, $2.1 \%$ of people have been reported to have COVID-19 [22]. The first wave of COVID-19 was finished at the beginning of our study, but the number of patients had increased by the end of the study [23]. The detection of all the positive cases in the last week of the study suggests an increasing number of asymptomatic cases.

Although the data related to COVID-19 cases in the early gestational weeks are limited, the SARS-CoV-2 infection has not been associated with spontaneous abortion $[24,25]$. In our study, none of the miscarriage patients had a SARS-CoV-2 infection. The sensitivity of antibody tests is too low. IgM was detected in 23 percent and Ig G was 30 percent in the first seven days after symptom onset in patients with COVID-19 [26]. It has been shown that the IgG antibody count of $40 \%$ of asymptomatic cases is reset in eight weeks after infection [27]. In this respect, asymptomatic cases in both term PROM and miscarriage pregnancies may not be detected due to either the early period of the disease or the passage of time. The most rational approach seems to be to create regular screening programs in high-risk patients, such as pregnant women.

With the COVID-19 pandemic, depression and anxiety significantly increase in the pregnant population, and researchers recommend that all pregnant women should receive psychosocial support during this period [28, 29]. Miscarriage was associated with increased cortisol and decreased immunity as a result of increased acute and chronic stress [30-32]. Changing social balance and economic concerns during the pandemic process can be a major stressor for patients with low socioeconomic status, who make up the main segment of patients at our hospital. Above $\% 90$ of patients thought SARS-CoV-2 would infect themselves and their children. The number of patients who would not consider pregnancy if they were not pregnant during this period was significantly higher in term PROM (48.3\%) compared to miscarriage $(27.8 \%)(p<0.005)$ patients. Since they are exposed to the COVID-19 pandemic process longer, anxiety may be more in the advanced weeks of pregnancy.
The strength of the study is the high number of patient groups. The main limitation is that not all the patients could be screened.

\section{CONCLUSIONS}

No COVID-19 cases were detected in the asymptomatic miscarriage patients. In the asymptomatic term PROM patients, the effects of COVID were not observed. Although COVID-19 did not show its direct effects in this study, intermittently screening all pregnant women to determine the effects of COVID-19 on pregnant women seems to be the most rational approach.

\section{Conflicts of interest}

The authors have no conflicts of interest to declare.

\section{Funding}

None.

\section{REFERENCES}

1. Lu R, Zhao X, Li J, et al. Genomic characterisation and epidemiology of 2019 novel coronavirus: implications for virus origins and receptor binding. Lancet. 2020; 395(10224): 565-574, doi: 10.1016/S01406736(20)30251-8, indexed in Pubmed: 32007145.

2. World Health Organization, Coronavirus disease (COVID-2019) situation reports 2020. https://www.who.int/emergencies/diseases/novel-coronavirus-2019/situation-reports (1.09.2020).

3. General directorate of public health, COVID-19 (SARS-CoV-2 infection) guideline Scientific Committee Report 2020. https://covid19bilgi.saglik. gov.tr/depo/rehberler/COVID-19_Rehberi.pdf?type=file (1.12.2020).

4. Czeresnia RM, Trad AT, Britto IS, et al. SARS-CoV-2 and Pregnancy: A Review of the Facts. Rev Bras Ginecol Obstet. 2020; 42(9): 562-568, doi: 10.1055/s-0040-1715137, indexed in Pubmed: 32992359.

5. Ellington S, Strid P, Tong VT, et al. Characteristics of Women of Reproductive Age with Laboratory-Confirmed SARS-CoV-2 Infection by Pregnancy Status - United States, January 22-June 7, 2020. MMWR Morb Mortal Wkly Rep. 2020; 69(25): 769-775, doi: 10.15585/mmwr.mm6925a1, indexed in Pubmed: 32584795.

6. Elshafeey F, Magdi R, Hindi N, et al. A systematic scoping review of COVID-19 during pregnancy and childbirth. Int J Gynaecol Obstet. 2020; 150(1): 47-52, doi: 10.1002/ijgo.13182, indexed in Pubmed: 32330287.

7. Sutton D, Fuchs K, D'Alton M, et al. Universal Screening for SARS-CoV-2 in Women Admitted for Delivery. N Engl J Med. 2020; 382(22): 2163-2164, doi: 10.1056/NEJMc2009316, indexed in Pubmed: 32283004.

8. Breslin N, Baptiste C, Gyamfi-Bannerman C, et al. Coronavirus disease 2019 infection among asymptomatic and symptomatic pregnant women: two weeks of confirmed presentations to an affiliated pair of New York City hospitals. American Journal of Obstetrics \& Gynecology MFM. 2020; 2(2): 100118, doi: 10.1016/j.ajogmf.2020.100118.

9. Kennedy DM, Zambrano GJ, Wang Y, et al. Modeling the effects of intervention strategies on COVID-19 transmission dynamics. J Clin Virol. 2020; 128: 104440, doi: 10.1016/j.jcv.2020.104440, indexed in Pubmed: 32425658.

10. Yan J, Guo J, Fan C, et al. Coronavirus disease 2019 in pregnant women: a report based on 116 cases. Am J Obstet Gynecol. 2020;223(1): 111.e1111.e14, doi: 10.1016/j.ajog.2020.04.014, indexed in Pubmed: 32335053.

11. Kirtsman M, Diambomba $Y$, Poutanen SM, et al. Probable congenital SARS-CoV-2 infection in a neonate born to a woman with active SARS-CoV-2 infection. CMAJ. 2020; 192(24): E647-E650, doi: 10.1503/cmaj.200821, indexed in Pubmed: 32409520.

12. Vivanti AJ, Vauloup-Fellous $C$, Prevot $S$, et al. Transplacental transmission of SARS-CoV-2 infection. Nat Commun. 2020; 11(1): 3572, doi: 10.1038/s41467-020-17436-6, indexed in Pubmed: 32665677.

13. Kotlyar AM, Grechukhina O, Chen A, et al. Vertical transmission of coronavirus disease 2019: a systematic review and meta-analysis. Am 
J Obstet Gynecol. 2021; 224(1):35-53.e3, doi: 10.1016/j.ajog.2020.07.049, indexed in Pubmed: 32739398.

14. Huntley BJF, Huntley ES, Di Mascio D, et al. Rates of Maternal and Perinatal Mortality and Vertical Transmission in Pregnancies Complicated by Severe Acute Respiratory Syndrome Coronavirus 2 (SARS-Co-V-2) Infection: A Systematic Review. Obstet Gynecol. 2020; 136(2): 303-312, doi: 10.1097/AOG.0000000000004010, indexed in Pubmed: 32516273.

15. CELIK OY, OZKAN S, ERDOGAN K, et al. Did Pregnancy Complications Increase During The COVID-19 Pandemic Period? Medical Records. 2020, doi: 10.37990/medr.798767.

16. Campbell KH, Tornatore JM, Lawrence KE, et al. Prevalence of SARS-CoV-2 Among Patients Admitted for Childbirth in Southern Connecticut. JAMA. 2020; 323(24): 2520-2522, doi: 10.1001/jama.2020.8904, indexed in Pubmed: 32453390

17. Goldfarb IT, Diouf K, Barth WH, et al. Universal SARS-CoV-2 testing on admission to the labor and delivery unit: Low prevalence among asymptomatic obstetric patients. Infect Control Hosp Epidemiol. 2020; 41(9): 1095-1096, doi: 10.1017/ice.2020.255, indexed in Pubmed: 32456729.

18. Ai T, Yang Z, Hou H, et al. Correlation of Chest CT and RT-PCR Testing for Coronavirus Disease 2019 (COVID-19) in China: A Report of 1014 Cases. Radiology. 2020; 296(2):E32-E40, doi: 10.1148/radiol.2020200642, indexed in Pubmed: 32101510.

19. London V, McLaren R, Atallah F, et al. The Relationship between Status at Presentation and Outcomes among Pregnant Women with COVID-19. Am J Perinatol. 2020; 37(10): 991-994, doi: 10.1055/s-0040-1712164, indexed in Pubmed: 32428964.

20. Huang $C$, Wang $Y, L i X$, et al. Clinical features of patients infected with 2019 novel coronavirus in Wuhan, China. The Lancet. 2020; 395(10223): 497-506, doi: 10.1016/s0140-6736(20)30183-5.

21. Kumar D, Moore RM, Mercer BM, et al. The physiology of fetal membrane weakening and rupture: Insights gained from the determination of physical properties revisited. Placenta. 2016; 42: 59-73, doi: 10.1016/j. placenta.2016.03.015, indexed in Pubmed: 27238715.

22. COVID-19 Situation Report Turkey 2020. https://sbsgm.saglik.gov. tr/TR,66424/covid-19-situation-report-turkey.html (10.12.2020).
23. General Coronavirus Table. https://covid19.saglik.gov.tr/EN-69532/general-coronavirus-table.html (1.08.2020).

24. Elshafeey F, Magdi $R$, Hindi N, et al. A systematic scoping review of COVID-19 during pregnancy and childbirth. Int J Gynaecol Obstet. 2020; 150(1): 47-52, doi: 10.1002/ijgo.13182, indexed in Pubmed: 32330287.

25. Yan J, Guo J, Fan C, et al. Coronavirus disease 2019 in pregnant women: a report based on 116 cases. Am J Obstet Gynecol. 2020; 223(1): 111.e1111.e14, doi: 10.1016/j.ajog.2020.04.014, indexed in Pubmed: 32335053.

26. Deeks JJ, Dinnes J, Takwoingi Y, et al. Cochrane COVID-19 Diagnostic Test Accuracy Group. Antibody tests for identification of current and past infection with SARS-CoV-2. Cochrane Database Syst Rev. 2020;6:CD013652, doi: 10.1002/14651858.CD013652, indexed in Pubmed: 32584464.

27. Long QX, Tang XJ, Shi QL, et al. Clinical and immunological assessment of asymptomatic SARS-CoV-2 infections. Nat Med. 2020; 26(8): 1200-1204, doi: 10.1038/s41591-020-0965-6, indexed in Pubmed: 32555424.

28. Durankuş F, Aksu E. Effects of the COVID-19 pandemic on anxiety and depressive symptoms in pregnant women: a preliminary study. J Matern Fetal Neonatal Med. 2020 [Epub ahead of print]: 1-7, doi: 10.1080/14767058.2020.1763946, indexed in Pubmed: 32419558.

29. Sinaci S, Ozden Tokalioglu E, Ocal D, et al. Does having a high-risk pregnancy influence anxiety level during the COVID-19 pandemic? Eur J Obstet Gynecol Reprod Biol. 2020; 255: 190-196, doi: 10.1016/j. ejogrb.2020.10.055, indexed in Pubmed: 33147531.

30. Qu F, Wu Y, Zhu YH, et al. The association between psychological stress and miscarriage: A systematic review and meta-analysis. Sci Rep. 2017; 7(1): 1731, doi: 10.1038/s41598-017-01792-3, indexed in Pubmed: 28496110.

31. Frazier T, Hogue $\mathrm{CJ}$, Bonney EA, et al. Weathering the storm; a review of pre-pregnancy stress and risk of spontaneous abortion. Psychoneuroendocrinology. 2018; 92: 142-154, doi: 10.1016/j.psyneuen.2018.03.001, indexed in Pubmed: 29628283.

32. Nepomnaschy PA, Welch KB, McConnell DS, et al. Cortisol levels and very early pregnancy loss in humans. Proc Natl Acad Sci U S A. 2006; 103(10): 3938-3942, doi: 10.1073/pnas.0511183103, indexed in Pubmed: 16495411. 